\title{
Community-Based Stroke Rehabilitation: Recovery continued?
}

The term 'neuroplasticity' first appeared in the research literature in 1973 in the study of visual system regeneration in animals. ${ }^{1}$ Twenty years later Dobkin discussed neuroplasticity and its role in recovery after stroke, ${ }^{2}$ which began to dispel the long held view that, after brain swelling and inflammation subsided, there was no further capacity for stroke recovery. Although the most substantial improvements occur within the first 30 days poststroke, we now understand that further spontaneous recovery can be seen up to about 90 days post-stroke. ${ }^{3}$ With sufficient intensity, some rehabilitation treatments such as constraint-induced movement therapy (CIMT) result in further plasticity and recovery beyond the typical "window of recovery'. 4 To what extent ongoing recovery occurs after discharge from inpatient rehabilitative care is still under debate. ${ }^{5}$ The study "Community Stroke Rehabilitation Teams: Providing Home-Based Rehabilitation in Ontario Canada", by Allen et l, $^{6}$ for the first time examines quantitative outcomes at admission, discharge and follow-up in a large cohort of people receiving Community-Based Stroke Rehabilitation (CBSR) in Canada. The study raises some important issues in community-based stroke care: namely: 1. Are the improvements meaningful (clinically-important) for the patient and family? 2. Do the results suggest that the improvements are due to compensatory use of the less affected side or true recovery of the affected side? 3 . What are the active ingredients? 4. Is this typical of community stroke care in Canada or is this an exemplary service?

In order to examine the results of Allen et $\mathrm{al}^{6}$ in context of stroke recovery in Canada, data from the Canadian Institutes for Health Information (CIHI) National Rehabilitation Reporting System $^{7}$ was accessed for the year 2011-12, about the midpoint of the data collection in the CBSR study. The recovery plot modelled in Figure 1 shows that people admitted to inpatient stroke rehabilitation in Canada score on average about 76.6 on Functional Independence Measure (FIM). They improve to a score of about 99 over the course of 33 days. Although it is recognized that participants in the CBSR program were referred from several agencies (e.g. hospital, community), 50\% were referred from inpatient rehabilitation. Data from Allen et $\mathrm{al}^{6}$ show that, in CBSR, participants gained a further five FIM points on average (Table 3 in the article). Although this is a small gain in relation to total change, five additional points translates to care reduced from dependence to independence (score change from 2 to 7 ) on one item or reduced level of assistance, from moderate to minimal, on five items. As pointed out by the authors, the change was most likely clinically meaningful and important for the caregiver. In fact, in the Caregiver Assistance subcomponent of the Caregiver Assistance and Confidence Scale, ${ }^{8}$ level of assistance dropped from 39.96 to 32.1 at discharge and then to 28.82 at follow-up; a change of about 11 points out of a possible 102 (Table 3 Allen et $\mathrm{al}^{6}$ ).

It is important to determine whether or not CBSR improves neurological impairment or promotes compensation since a 'function-only' approach without attention to neurological impairments could hinder future gains in quality of life. ${ }^{9}$ If improvement in activities of daily living (ADL) were due primarily to overuse of the stronger side, one would expect to see an improvement in ADL without corresponding improvement in the affected arm and leg. Although the study by Allen et al. does not include outcomes at the impairment level, participants reported the function of their affected side using the Stroke Impact Scale (SIS). ${ }^{10}$ SIS data (Table 3 in the article) showed that improvement in ADL (8.8 points) was accompanied by improvement in strength of the affected side ( 8.2 points) and affected hand strength (11.3 points). These findings support the premise that, with intervention, there is true recovery continuing to occur on the affected side post-hospital discharge. In order to advance this field forward, other regions with CBSR should decide on appropriate performance measures and consistently enter the data and the details of the interventions into a database. Future databases should include objective tools that measure outcome at the impairment level, such as grip strength, gait parameters or cognition, in order to determine if recovery represents neurobiological repair or compensation. ${ }^{11}$

Canadian Stroke Best Practices Guidelines clearly outline the evidence that supports coordinated, specialized inpatient rehabilitative care followed by similarly specialized outpatient or community care. ${ }^{12}$ The earliest systematic review and metaanalysis of $\mathrm{CBSR}^{13}$ focused on preventing post-discharge deterioration and reported that death and deterioration was significantly less for patients receiving therapy-based community stroke care (OR 0.67). A more recent review of 14 randomized controlled trials ${ }^{14}$ showed that in terms of further recovery, there was no effect of community-based intervention. However, in this review only three of the programs included rehabilitation therapy interventions (as opposed to assessment and education). The 'active ingredients' of optimal CBSR are not known. In an article by Walker et $\mathrm{al}^{5}$ the authors asked, with regard to intensity of community-based stroke programs, "How much therapy is enough in practice and how many staff, and at what skill level, are required to provide it?" The program evaluated by Allen et $\mathrm{al}^{6}$ provided modest staff resources at 2.3 visit per week (33 visits) over four months. Most interventions were provided by a rehabilitation therapist under the direction of occupational therapists, speech language pathologists and physiotherapists suggesting that most treatment involved rehabilitation therapy. These findings support previous reviews that favour therapy-based interventions in the community rather than home assessments or education.

In Canada, we know that only $19 \%$ of people admitted to acute care with a stroke are transferred to inpatient rehabilitation. Of those who do receive rehabilitation, $38 \%$ are discharged home with some services; the remainder discharged without any services or to long term care. ${ }^{15}$ The administration of health care in Canada is directed by the provinces and provincial health boards so it is not surprising that community-based rehabilitative care availability and intensity vary widely. In some provinces, 


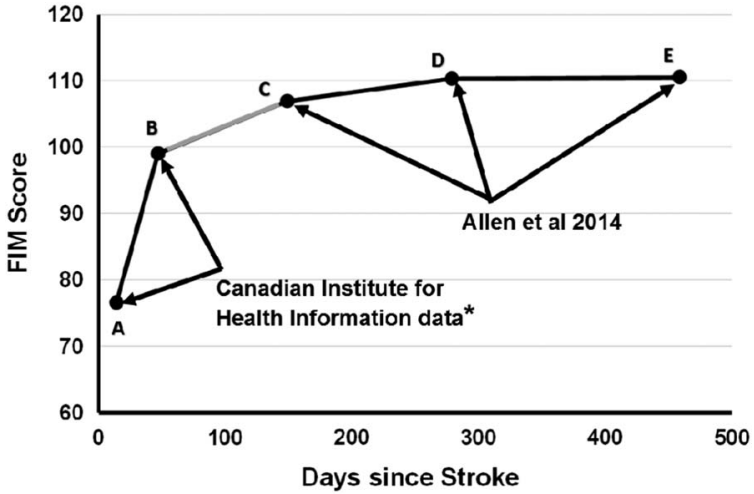

Figure 1: Estimated FIM Score at key points in stroke care: Admission $(A)$ and discharge (B) from inpatient rehabilitation and admission $(C)$, discharge $(D)$ and 6 month follow-up (E) from CBSR.* Data derived from Canadian Institute for Health Information National Rehabilitation Reporting System ${ }^{7}$ reporting year 2011-12.

community-access centres and extramural hospitals provide rehabilitation whereas in other areas there are almost no community-based rehabilitation services. Unfortunately for Canadians with stroke living outside of Southwestern Ontario, the program studied by Allen et al is likely an exemplary service. However it could provide a potential template for practice and measurement of outcomes of these exemplary programs are key to advocating for them. Country-wide consensus that recommends a minimal dataset for CSBR is needed; as expressed by Lord Kelvin (1883), "You can't fix what you can't measure". Although a randomized controlled trial of CBSR is desirable, providing a comparison group would be challenging. A trial would likely involve comparing two intensities or structures of CBSR or comparing outcomes from two jurisdictions. Future CBSR studies should also incorporate economic evaluation.

In summary, the study of CBSR by Allen and group ${ }^{6}$ strengthens the notion that there is continued meaningful recovery available with sufficiently intense therapy-based interventions in the chronic phase of stroke. A great deal of research and health resources have been expended in acute and hyperacute care of stroke. ${ }^{16}$ Since there are more stroke survivors than ever before and since CBSR is relatively inexpensive, community-based therapies provide an economical method to maximize gains. Health decision-makers in Canada would be wise to examine and invest in community-based rehabilitation.

\section{DisClosures}

The author reports no disclosures.

\author{
Michelle Ploughman \\ Physical Medicine \& Rehabilitation, Faculty of Medicine \\ Recovery \& Performance Laboratory, Memorial University, \\ St John's, Newfoundland, Canada \\ Email: Michelle.Ploughman@med.mun.ca
}

\section{REFERENCES}

1. Meyer RL, Sperry RW. Tests for neuroplasticity in the anuran retinotectal system. Exp Neurol. 1973;40(2):525-39.

2. Dobkin BH. Neuroplasticity. Key to recovery after central nervous system injury. West J Med. 1993;159(1):56-60.

3. Wahl AS, Schwab ME. Finding an optimal rehabilitation paradigm after stroke: enhancing fiber growth and training of the brain at the right moment. Front Human Neurosci. 2014;8:381.

4. Wolf SL, Winstein CJ, Miller JP, et al. Effect of constraint-induced movement therapy on upper extremity function 3 to 9 months after stroke: the EXCITE randomized clinical trial. JAMA. 2006;296(17):2095-104.

5. Walker MF, Sunnerhagen KS, Fisher RJ. Evidence-based community stroke rehabilitation. Stroke. 2013;44(1):293-7.

6. Allen L, Richardson M, McIntyre A, et al. Community stroke rehabilitation teams: Providing home-based stroke rehabilitation in Ontario, Canada. Can J Neurol Sci. 2014;41:697-703.

7. Canadian Institute for Health Information. National Rehabilitation Reporting System. 2011. http://www.cihi.ca/CIHI-ext-portal/ internet/EN/Quick_Stats/quick+stats/quick_stats_main?xTopic = Hospital\%20Care\&pageNumber $=3 \&$ resultCount $=10 \&$ filterType $\mathrm{By}=$ undefined\&filterTopicBy $=5$ \&autorefresh $=1$

8. Cameron JI, Franche RL, Cheung AM, Stewart DE. Lifestyle interference and emotional distress in family caregivers of advanced cancer patients. Cancer. 2002;94(2):521-7.

9. Mayo NE, Scott SC, Bayley M, et al. Modeling health-related quality of life in people recovering from stroke. Qual Life Research. 2013: DOI 10.1007/s11136-013-0605-4

10. Duncan PW, Wallace D, Lai SM, Johnson D, Embretson S, Laster LJ. The stroke impact scale version 2.0. Evaluation of reliability, validity, and sensitivity to change. Stroke. 1999;30(10):2131-40.

11. Krakauer JW, Carmichael ST, Corbett D, Wittenberg GF. Getting neurorehabilitation right: what can be learned from animal models? Neurorehab Neural Repair. 2012;26(8):923-31.

12. Heart and Stroke Foundation of Canada. Canadian Stroke Best Practices Recommendations; 2013. http://www.strokebestpractices. ca/index.php/stroke-rehabilitation/

13. Outpatient Service Trialists. Therapy-based rehabilitation services for stroke patients at home. Cochrane Database of Systematic Reviews. 2003;(1):CD002925.

14. Fens M, Vluggen T, van Haastregt JC, Verbunt JA, Beusmans GH, van Heugten CM. Multidisciplinary care for stroke patients living in the community: a systematic review. J Rehab Med. 2013; 45(4):321-30.

15. Canadian Stroke Network. The quality of stroke care in Canada 2011. http://canadianstrokenetwork.ca/en/service/quality-of-stroke-carein-canada-2011/

16. Teasell R, Hussein N, McClure A, Meyer M. Stroke: More than a 'brain attack'. Int J Stroke. 2014;9(2):188-90. 